\title{
MEDIACIÓN GENERADORA DE CAPITAL SOCIAL
}

\author{
Mediation Generating Social capital
}

Francisco GORJÓN ${ }^{1}$

Francisco BARRERA ${ }^{2}$

\begin{abstract}
Sumario:
I. Introducción; II. Contexto del capital social ante los MASC; III. Concepto de capital social; IV. El capital social intangible de la mediación; V. El capital social como cultura; VI. Fuentes consultadas
\end{abstract}

Resumen: La mediación es considerada actualmente un factor de desarrollo, un factor de modernización y de internacionalización del sistema de administración y procuración de justicia, sin embargo, no había sido considerada como generadora de capital social, esta tesis surge en razón de los elementos coincidentes entre ambas figuras de impacto social, al grado de incidir analógicamente en sus intangibles, al igual que en su misión, mientras la mediación procura la convivencia pacifica y alienta el desarrollo, el capital social, contiene los mismos elementos y la misma misión, bajo la premisa de la participación ciudadana.

Palabras Clave: Capital social, mediación, métodos alternos de solución de conflictos, cultura, intangibles del capital social.

Abstract: Mediation is now considered a development factor, a factor of modernization and internationalization of the system of administration and law enforcement, however, had not been considered as a generator of social capital, this idea arises because of the matching elements between the two figures of social impact, the degree of influence analogically intangible, like its mission, while mediation seeks peaceful coexistence and encourages development, social capital, contains the same elements and the same mission, under the premise of citizen participation.

Keyword: Social capital, mediation, alternative dispute resolution methods, culture, intangible social capital.

\section{INTRODUCCIÓN}

La reforma procesal constitucional penal ha generado una revolución en todo nuestro sistema jurídico, transformando el actual sistema hacia un sistema acusatorio, que ha trastocado el hacer cotidiano de los abogados y de todos los usuarios y prestadores de servicios del sistema de impartición y procuración de justicia. Introduciendo dinámicas diversas en el proceso, como lo es la oralidad, la transparencia, la argumentación, la prontitud, la expedites, el

\footnotetext{
${ }^{1}$ Director académico del Doctorado de Métodos Alternos de Solución de Conflictos de la Universidad Autónoma de Nuevo León

${ }^{2}$ Director de Innovación de la Universidad Autónoma de Nuevo León.
} 
rol del ministerio público o fiscal en el proceso, el del juez en diferentes estadios y el papel preponderante de la ciudadanía a través de la mediación, ya que no se puede considerar la existencia del sistema acusatorio sin las salidas alternas, que tienen un rango especial en el Código Penal y en la novísima Ley Nacional de Mecanismos Alternativos de Solución de Controversias en Materia Penal y no solo estas, sino que actualmente se encuentran vigentes más de 26 leyes de justicia alternativa y 15 constituciones locales prevén la solución por esta vía como una garantía, generándose entonces toda una política pública en favor de la implementación de la mediación y de los demás métodos alternos de solución de conflictos, con el afán de generar una cultura del uso de los métodos alternos y en consecuencia ciudadanizar la justicia.

Esto nos obliga a ver el derecho desde otra perspectiva, al darle un sentido más social e interrelacionando diversos sistemas como el retributivo, con el alternativo y con el restaurativo, este último también presente en la Ley Nacional de Mecanismos Alternativos, a través de sus juntas restaurativas. Generando un efecto de transdiciplinariedad en la solución de conflictos de diversa índole, ya sea penal, civil, familiar, administrativo, entre otros.

Esta idea de socializar el derecho nos obliga a pensar que a través de la mediación se le otorgan nuevos atributos al derecho, ya no solo como el garante de la sociedad o el macro regulador del estado de derecho, sino como un generador de capital social, que contribuye evidentemente al desarrollo social, al equilibro del bienestar y al buen vivir en condiciones socialmente aceptables conforme a parámetros internacionales en un sistema económico social como el nuestro. La norma ya no será más solo una guía o una regla a seguir, será un reactivo conductor de calidad de vida y en consecuencia generadora de capital social a través de la mediación, en el momento de resolver el conflicto y de sus evidentes favorables consecuencias.

\section{CONTEXTO DEL CAPITAL SOCIAL ANTE LOS MASC}

La mediación es una ciencia social emergente, y como tal sus implicaciones científicas son patentes en cuanto a sus áreas de incidencia, de ahí de entender al capital social como un arquetipo de la ciencia de la mediación como uno de los principales indicadores del constructo de la cientificidad ${ }^{3} \mathrm{y}$ un área de incidencia efectiva, que por lo tanto una influye sobre otra, al respecto Chaparro ${ }^{4}$ señala que las revoluciones científicas actuales están generando nuevas formas de organización social, por lo que esta idea incide en la vocación de los métodos alternos, en especial en la mediación, ya que su instrumentalización genera nuevas condición de vida de las personas, y en consecuencia el uso de nuevas metodologías de interacción o de administración o generación de conocimiento que producen capital social', principalmente cuando de gestión del conflicto se trata. Centrándose estos nuevos esquemas asociativos en la cooperación, que si visualizamos la esencia de la mediación que es la voluntad de las partes implica la cooperación, será entonces el mismo principio o motor de acción

\footnotetext{
${ }^{3}$ GORJÓN GÓMEZ, Francisco Javier. "De la instrumentalización a la cientificidad de la mediación y de los métodos alternos de solución de conflictos". En GORJÓN, Francisco y PESQUEIRA, Jorge. La ciencia de la mediación, ed. Tirant Lo Blanch, México2015. Pp. 13-32.

${ }^{4}$ CHAPARRO, F. "Conocimiento, aprendizaje y capital social como motor de desarrollo" Ci.Inf. Brasilia, 2001. pp 19-31.

5 Idem.
} 
de la evolución del derecho, de la sociedad del conocimiento y en consecuencia del capital social, ya que están simbióticamente asociados.

Por lo tanto, consideramos determinante entender el concepto de capital social así como sus características y diversos elementos operativos, pero, primero vincularlo a los métodos alternos y dos, determinar que su desarrollo produce en interacción con la mediación un cambio social, modernización del derecho, socialización de la norma y por consecuencia un capital social fincado en el resultado del procedimiento de solución del conflicto, traducido este en un acuerdo de método alterno o de mediación vinculante y con reconocimiento normativo, con un impacto revitalizador y este a su vez se ve materializado en un acuerdo generador de capital social, que como señalaremos más adelante, ambos conceptos son provocadores de desarrollo, de cultura y de bienestar particular o social.

\section{CONCEPTO CAPITAL SOCIAL}

Señalar al capital social es referencias de modernidad, es pensar en un mejor funcionamiento o desempeño organizacional, basado según Montoya ${ }^{6}$ en cuatro dimensiones, integridad, autonomía, integración y sinergia. La primera de ellas la interpretamos como el accionar de un todo de forma ordenada conforme a principios generales operativos aceptados por la comunidad en general como sucede con la norma, según sus usos y costumbres ósea una cultura; autonomía es el accionar sobre este proceso integrador de políticas altamente sociales que operan con un mismo sentido como sucede con los métodos alternos en especial la mediación; integración que significa su impacto de sumar voluntades en pro de una misma acción de resultados esperados favorables de bien común igualmente fin último de la mediación, y por último la sinergia que es el encausamiento de esas voluntades de forma proactiva en pro de un beneficio común, acuerdos.

Conforme estas dimensiones podemos entender al capital social según Woolcock y Nayaran $^{7}$ como el actuar de la gente de forma colectiva, basándose en la confianza y la reciprocidad en un marco de igualdad, generando un trato social equilibrado entre desiguales, esto es un sistema de bienestar social, conformado de igual manera por el nivel de confianza entre los actores sociales, entre las normas de comportamiento cívico y su nivel de asociatividad, fortaleciendo el tejido social como una fortaleza, es esto en esencia el capital social.

El capital social produce confianza y actúa como un ahorrador de conflictos potenciales, limitando el pleitismo, generado en consecuencia actitudes positivas, contribuyendo al bienestar social, según describe Kliksberg, ${ }^{8}$ esta idea que acuña nuestro autor es talvez uno de los centros de la gestión del conflicto, los evitamos, los solucionamos, empero aprovechamos todos sus elementos, para generar evolución social, es por eso que la solución del conflicto ya no se entiende como tal, se entiende como gestión del conflicto, la mediación es el tamiz que lo logra, y como bien coinciden algunos autores expertos en la gestión del conflicto como Ferré Salvá ${ }^{9}$ evitar el pleitismo, genera actitudes positivas y genera bienestar social, al

\footnotetext{
${ }^{6}$ MONTOYA, E. “El concepto de capital social y su utilidad para el análisis de dinámicas de desarrollo”, Revista de Fomento Social, Córdoba, 2001, pp. 35-63.

7 WOOLCOCK, M. y NAYARAN, D. Capital social: implicaciones para la teoría, la investigación y las políticas de desarrollo. Banco Mundial, 2013, pp. 4-24.

8 KLIKSBERG, Bernardo. "Capital social y cultura en el proceso de desarrollo”, Revista Venezolana de Gerencia, 1999, pp. 11-50.

9 FERRÈ SALVÁ, S. Gestión de conflictos: taller de mediación. Un enfoque socio efectivo, ed. Ariel, Barcelona, 2006. pp. 13-45.
} 
igual que perdón, si la sociedad no es capaz de perdonarse se queda estática, y el perdón es movimiento, el perdón es reconciliación, el perdón en un valor positivo, es un intangible de toda sociedad que se jacte de ser pacífica1o y principalmente de los métodos alternos.

Esta misma idea se encuentra en el imaginario de los métodos alternos en especial en el de la mediación, como generadora de puentes basado en la confianza, como veremos en próximo epígrafe de los intangibles del capital social, entiéndanse estos como la apreciación positiva de los efectos del capital social en el modus vivendi de las personas, en razón de la teoría del valor intangible de los métodos alternos de solución de conflictos.

\section{EL CAPITAL SOCIAL INTANGIBLE DE LA MEDIACIÓN}

Afirmamos de igual manera que el capital social podemos constituirlo como un intangible de la mediación, así como de los demás métodos alternos de solución de conflictos, según Gorjón ${ }^{11}$ es necesario visualizar a los métodos alternos desde una perspectiva positiva, ya que actualmente este proceso no se da, la sociedad los ve desde diversas ópticas, entre ellas la positiva, sin embargo, prevalecen con mayor influencia aquellas que generan confusión, entendiendo esto que la mediación es vista desde la experiencia de otras ciencias, por ello se encuentra presente el efecto de la judicialización de los MASC, claro ejemplo de la reproducción de patrones de otras ciencias en la nuestra.

La ciencia de la mediación establece dos constructos, el de la instrumentalización y el de la cientificidad, en ambos podríamos decir que el capital social se encuentra presente, en el primero identificado como efecto de la aplicación de la medicación y en el segundo lo identificamos como indicador puntual como intangible, generando con ello una innovación, es decir dentro de la dimensión de la mediación como objeto de estudio y de la dimensión de la mediación como profesión.

Es importante considerar al capital social como un esquema de innovación dentro de los MASC, ya que el sentido de este es generar condición sociales que antes no se habían considerado como observables dentro del devenir social, sus efectos según Durston ${ }^{12}$ Generan cambios, generan condiciones no previstas en la organización de un grupo social determinado, por ello cuando se identifica elementos del capital social, identificamos cambios y por lo regular positivos, ya que su efecto tiene ese sentido, si es capital social es bienestar, cuando este último elemento no está presente entonces no es capital social.

Diversos autores destacan las características y beneficios del capital social en acción, quiero decir operativamente hablando, entre ellos por mencionar alguno $\operatorname{Putman}^{13}$ que es considerado una de los autores más representativos del estudio del capital social, en su obra y a lo largo de ella hemos identificado diversos rasgos que coinciden con los intangibles de los

\footnotetext{
${ }^{10}$ VYNIAMATA, Eduardo. Conflictología, ed, Ariel, Barcelona ,2006. pp. 49-75.

${ }^{11}$ GORJÓN GÓMEZ, Francisco Javier. "El valor intangible de los métodos alternos de solución de conflictos. Estrategia de culturización de los MASC en el marco de la reforma procesal constitucional penal". Nuevo Sistema de Justicia Penal. México, 2013, pp. 50-63.

${ }^{12}$ DURSTON, J. "Construyendo capital social comunitario", Revista de la CEPAL 69, México, 1999, pp. 103119.

${ }^{13}$ PUTMAN, R.D. El declive del capital social. Un estudio internacional sobre las sociedades y el sentido común, ed. Círculo de lectores, Barcelona, 2003.
} 
MASC, ${ }^{14}$ empero que han sido tratados como características , elementos o principios del capital social, que bien identificamos como intangibles, a saber:

- El capital social genera vínculos fuertes entre la sociedad que genera redes y en consecuencia alianzas que dan paso a la normativización con impacto social;

- Genera reciprocidad entre las personas por la tanto eleva los niveles del equilibro entre las personas sociales y sus clases sociales; ${ }^{15}$

- Impulsa la confianza como primer elemento generador del vínculo entre las personas, no podríamos hablar de generación de vínculos o de reciprocidad sin la confianza entre las personas; ${ }^{16}$

- Facilita la vida en común, esto quiere decir que al encontrar punto de intersección entre las personas, su vida en común se mejora y se facilita, esto es logra que las personas se encuentre el nivel de entendimiento de sus interés y no de sus posiciones como suele ocurrir y es un esquema en el cual trabaja las acción de la gestión de los conflictos, visualizar los intereses y no las posiciones, factor de éxito de toda mediación y en consecuencia del capital intelectual;

- Fortalece la identidad, que es una sociedad sin identidad, nada, podría afirmarse, el capital social la genera como elemento de cohesión, podría pensarse en un súper pegamento, que hace a las personas unirse en pro de un mismo interés, como lo mencionamos hace un momento, si nos das el elemento de la identidad, nos permite ver al otro en el mismo rango, aunque sus interés y los míos sean diversos pero aun así coincidentes;

- Genera solidaridad, esto es que al momento de contar con una identidad, de tener interés comunes, de facilitar la vida en común, es lógico que se genera la solidaridad, esta no estaría presente si las posiciones de las partes siempre estuvieran presentes, empero cuando de interés comunes hablamos, el efecto solidario es más simple;

- Crea puentes, es este tal vez el intangible más importante de hasta los ahora mencionados, ya que si afirmamos que el capital social produce solidaridad, fortalece la identidad, facilita la vida en común, genera confianza, provoca redes en un entorno de reciprocidad, como sería esto factible sin puentes, al respecto siguiendo a Lederach17 en su obra afirma reiterativamente que para que una sociedad sea considerada pacifica es necesario la existencia de puentes entre ellos, esta teoría, es tan contunden-

\footnotetext{
${ }^{14}$ GORJÓN GÓMEZ, Francisco Javier. "El valor intangible de los métodos alternos de solución de conflictos. Estrategia de culturización de los MASC en el marco de la reforma procesal constitucional penal". Nuevo Sistema de Justicia Penal. México, 2013, pp. 50-63.

${ }^{15}$ HERREROS VAZQUEZ, F. “ ¿Son las relaciones sociales una fuente de recurso? Una definición de capital social”, Papers 67, Barcelona, 2002, pp. 129-148.

${ }^{16}$ SANCHEZ SANTOS, J.M. y PENA LOPEZ, J.A. "Actividad asociativa, confianza y generación de capital social: evidencia empírica”. EKONOMIAZ, San Sebastián, 2005, pp. 136-160.

${ }^{17}$ LEDERERACH, J.P. Building Peace. Susteinable reconciliation in divided societies. ed. United States Institute of Peace Press, Washington, 1997.
} 
te, que para que se dé un proceso de paz son necesarios los puentes, empero esta dinámica no solo es aplicable a sociedades enormemente conflictuadas, sino que también es aplicable y factible a relaciones micro, entre personas, sin puentes no hay comunicación y para construirlos se requiere un esfuerzo grupal, empero lo más importante de esto es que no basta de un esfuerzo de grupo, se requiere de una gran coordinación, ya que un puente no se construye por el centro, se construye desde las orillas, más aun cuando esa coordinación puede darse entre miembros de la misma comunidad o con personas fuera de nuestro entorno, desde mi perspectiva esta alegoría es la máxima de la sana convivencia, y de la vida con capital social.

- El capital social alienta que la sociedad enfrente sus problemas de forma compartida, ya que los mueve un mismo interés;

- Fomenta el entendimiento entre los miembros de la mismas comunidad, empero como señalamos anteriormente la construcción de puentes no solo puede darse entre los mismos miembros de una comunidad, sino que es necesario darse y interrelacionar con otros miembros de otras comunidades;

- Promueve la tolerancia, sin ella, los puentes no podrían construirse, el andamiaje de este seria débil, para que un puente de capital social sea fuerte es necesario la tolerancia, y esta se encuentra en los cimientos de toda edificación o sistema social exitoso, de larga vida, es un elemento sine qua non de la evolución social, no solo del capital social;

- Productividad, una sociedad moderna y exitosa requiere del ingrediente de la productividad, la simbiosis de desarrollo y del capital social es la productividad, se requieren elementos de confort, elementos económicos que le permiten a la sociedad allegarse de sus insumos no solo de supervivencia, sino insumos que le permitan crecer, evolucionar y que su modus vivendi sea más cómodo metafóricamente hablando, es cierto que el nivel económico no marca necesariamente a una sociedad pero si la incluye o las excluye de otras por su nivel, y su supervivencia es la fase inicial de su crecimiento; ${ }^{18}$

- Felicidad, cuando visualizamos una sociedad moderna, cuando la visualizamos exitosa, cuando la visualizamos pacifica, también debemos de considérala una sociedad feliz, y ello es también producto del capital social, al grado que la felicidad es actualmente considerada un indicador económico, empero, también es un indicador moral, es inalienable considerar una buena relación sin que esta produzca felicidad, la felicidad es producto del respeto, es cierto que su efecto filosófico, desborda este estudio, pero el concepto de felicidad unifica los nivel económicos sociales, que tal vez es más importante esta que el nivel económico en la que se encuentre, esto quiere decir que la felicidad es producto multifactorial de las relaciones entre personas viviendo en sociedad y no solo depende de un factor económico para lograrlo, actualmente existen

\footnotetext{
${ }^{18}$ SANCHEZ JIMENEZ, V. "El capital social como instrumento de análisis económico", Cuadernos de relaciones laborales. Madrid, 2012, pp. 473-493.
} 
estudios que buscan las sociedades más felices ${ }^{19}$ y esa comparación genera un benchmarking para otras que no se encuentran bajo las mismas condiciones;

- Efectividad es referente a las instituciones creadas en el entorno del desarrollo del capital social, son las operadoras de los diferentes sentidos y dimensiones que la dan vida y sentido de esta visión, si sus organizadoras o administradoras no son efectivas en el cumplimiento de sus diversos postulados y misiones, entonces todo esfuerzo comunitario será inocuo ya que la efectividad institucionales la maquinaria que hace posible, que todos estos intangibles se vean operativizados y en consecuencia logran la oxigenación y alimentación de la sociedad.

- Modernidad, visualizar en movimiento a la mediación como generadora de capital social es un acto de modernidad, ya que esta se deriva de prácticas exitosas en sistemas de solución de conflictos alternos en otros países y en consecuencia probados en su funcionamiento, los métodos alternos son un indicador de modernidad al ser parte evidente de la trasformación de un sistema como lo dejamos patentizado en la introducción de este articulo;

- Internacionalización, siguiendo la misma lógica que el intangible anterior no podemos concebir una sociedad aislada, su dinámica responde necesariamente a sinergias planetarias, el capital social es resultado de estas sinergias, como también lo es la implementación de la mediación y de los demás métodos alternos en la solución de conflictos sociales y en la concertación social.

- Si observamos detenidamente todos estos rasgos del capital social, podríamos estar presentes ante una futura clasificación de los intangibles del capital social, sería una innovación en el devenir del entendimiento de los métodos alternos de la búsqueda de factores que justifican día a día que la mediación es una ciencia socialmente emergente; que al igual que los intangibles de la mediación y de los MASC producen evolución y cultura en pro del concertación social y de la paz, fin último de una vida socialmente aceptable y de vida en común de las sociedad modernas.

\section{EL CAPITAL SOCIAL COMO CULTURA}

La generación de un estilo de vida implica un esfuerzo en conjunto, implica un desarrollo tamizado por un ideal, implica una cultura que incide entre diversos grupos sociales, que actúan en pro de un mismo ideal pero conforme a determinados intereses. La cultura según Kliksberg ${ }^{20}$ es un factor decisivo de cohesión social, por lo que las personas pueden reconocerse mutuamente, cultivarse, crecer en conjunto y desarrollar la autoestima colectiva traduciéndose un estilo de vida en una cultura y esta a su vez en valores, y estos en desarrollo.

El tema a pensar es la necesidad de la creación de condiciones adecuadas para impulsar el desarrollo, sin ellas no se concretara, es en este punto en donde entra en escena la mediación, ya que si repasamos algunos elementos del capital social antes señalados, como

\footnotetext{
${ }^{19}$ MANZILlA PRIETO, F. Ranking de Felicidad en México, ed. UPAEP, México, 2012.

${ }^{20}$ KLIKSBERG, Bernardo. "Capital social y cultura en el proceso de desarrollo", Revista Venezolana de Gerencia, 1999, pp. 11-50.
} 
la confianza, este concepto es relevante para la interacción humana y de igual manera base para lograr la voluntariedad, sin esta confianza y sin esta voluntad no hay forma de resolver ningún conflicto; en el mismo sentido observamos a la reciprocidad, señalábamos que la reciprocidad impacta en la generación de igualdad, no podemos concebir un procedimiento de mediación sin ese nivel de igualdad, aunando un poco más el capital social es un producto de las actitudes positivas, siendo este último talvez una de los intangibles más relevantes de la mediación y de los métodos alternos de solución de conflictos, mirar la gestión del conflicto positivamente, entender el valor de la mediación desde un enfoque positivo, no negativo, por lo tanto será un generador de cultura de aplicabilidad.

Sumamos de igual manera a esta descripción el esfuerzo conjunto, que es la ejemplificación del principio de autonomía de la voluntad de las partes que rigen indubitablemente a los métodos alternos y a la mediación, pero para que ello se dé, debe actuar la sociedad conforme a mismos interese, el principio de cohesión social aportado por el capital social y el principio resolutorio de la mediación es el mismo, por lo tanto, actuar en base a los intereses se convierte en un sistema de desarrollo, ya sea de forma colectiva o de forma particular. Esto nos lleva al último elemento que nos interesa que es el reconocimiento mutuo, si somos capaces de esto se generara las sinergias sociales y particulares de solución de conflictos pero también de convivencia social y en consecuencia de desarrollo.

Podemos afirmar que la mediación juega un papel preponderante en el desarrollo del capital social, independientemente del esquema cultural influenciado este por sistemas políticos o económicos, empero al ser ya un concepto de amplio espectro y reconocido planetariamente su aceptación es un hecho.

\section{FUENTES}

BIBLIOGRÁFICAS

CHAPARRO, F. "Conocimiento, aprendizaje y capital social como motor de desarrollo" Ci.Inf. Brasilia, 2001.

DURSTON, J. "Construyendo capital social comunitario", Revista de la CEPAL 69, México, 1999.

FERRÈ SALVÁ, S. Gestión de conflictos: taller de mediación. Un enfoque socio efectivo, ed. Ariel, Barcelona, 2006.

GORJÓN GÓMEZ, Francisco Javier. "De la instrumentalización a la cientificidad de la mediación y de los métodos alternos de solución de conflictos". En GORJÓN, Francisco y PESQUEIRA, Jorge. La ciencia de la mediación, ed. Tirant Lo Blanch, México2015.

, "El valor intangible de los métodos alternos de solución de conflictos. Estrategia de culturización de los MASC en el marco de la reforma procesal constitucional penal". Nuevo Sistema de Justicia Penal. México, 2013.

HERREROS VAZQUEZ, F. “'Son las relaciones sociales una fuente de recurso? Una definición de capital social”, Papers 67, Barcelona, 2002.

KLIKSBERG, Bernardo. "Capital social y cultura en el proceso de desarrollo", Revista Venezolana de Gerencia, 1999. 
LEDERERACH, J.P. Building Peace. Susteinable reconciliation in divided societies. ed. United States Institute of Peace Press, Washington, 1997.

MANZILLA PRIETO, F. RANKING DE FELICIDAD EN MÉXICO, ed. UPAEP, México, 2012.

MONTOYA, E. "El concepto de capital social y su utilidad para el análisis de dinámicas de desarrollo", Revista de Fomento Social, Córdoba, 2001.

PUTMAN, R.D. El declive del capital social. Un estudio internacional sobre las sociedades y el sentido común, ed. Círculo de lectores, Barcelona, 2003.

SANCHEZ JIMENEZ, V. "El capital social como instrumento de análisis económico”, Cuadernos de relaciones laborales. Madrid, 2012.

SANCHEZ SANTOS, J.M. y PENA LOPEZ, J.A. “Actividad asociativa, confianza y generación de capital social: evidencia empírica”. EKONOMIAZ, San Sebastián, 2005.

VYNIAMATA, Eduardo. Conflictología, ed, Ariel, Barcelona ,2006.

WOOLCOCK, M. y NAYARAN, D. Capital social: implicaciones para la teoría, la investigación y las políticas de desarrollo. Banco Mundial, 2013. 